\title{
Detection of immunoglobulin $\mathbf{E}$ using an aptamer based dot-blot assay
}

\author{
WANG YiXian, YE ZunZhong \& YING YiBin* \\ College of Biosystems Engineering and Food Science, Zhejiang University, Hangzhou 310058, China
}

Received September 15, 2012; accepted November 23, 2012; published online April 18, 2013

\begin{abstract}
A novel aptamer based dot-blot assay for the detection of immunoglobulin $\mathrm{E}$ ( $\operatorname{IgE}$ ) was developed. A biotinylated aptamer was employed as the bio-recognition element to specifically interact with the target protein immobilized onto a nitrocellulose membrane substrate. Avidin conjugated horseradish peroxidase was introduced onto the membrane through the biotin-avidin system to catalyze the hydrogen peroxide mediated oxidation of 3,3',5,5'-tetramethylbenzidine, thereby producing the blue-colored insoluble product. The intensity of the dots increased as the concentration of $\operatorname{IgE}$ increased. The spot intensity was quantified using a simple portable instrument. A linear response relationship between the spot intensity and the concentration of $\operatorname{IgE}$ over the range of 50 $\mathrm{nmol} / \mathrm{L}$ to $1 \mu \mathrm{mol} / \mathrm{L}$ was obtained. The detection limit for IgE using the aptamer-based assay was $2.89 \mathrm{nmol} / \mathrm{L}$. This assay was found to discriminate IgE from non-target proteins such as thrombin, bovine serum albumin and immunoglobulin G.
\end{abstract}

immunoglobulin E, dot-blot assay, aptamer, biotin-avidin system

Citation: Wang Y X, Ye Z Z, Ying Y B. Detection of immunoglobulin E using an aptamer based dot-blot assay. Chin Sci Bull, 2013, 58: 2938-2943, doi: $10.1007 / \mathrm{s} 11434-013-5702-9$

An allergic reaction can result in various autoimmune diseases, including atopic dermatitis, bronchial asthma, plant allergy, urticaria and eczema. In particular, atopic dermatitis is of particular interest because patient numbers have dramatically increased in recent years with over $10 \%$ of children worldwide thought to be suffering from an atopic related allergy [1,2]. Immunoglobulin E (IgE) key role in allergic reactions was discovered by Ishizaka et al. [3] and Johansson et al. [4]. IgE plays an important role in the allergic response, which is widely reported as a marker of atopic diseases, such as asthma, dermatitis and pollenosis [5]. As a result, rapid detection of the levels of IgE in serum samples is very important in dealing with patients that have allergy-mediated disorders. Traditional methods for the detection of IgE are the radioallergosorbent test (RAST) [6], the enzyme-linked immunosorbent assay (ELISA) and the dot-blot ELISA [7].

The dot-blot ELISA is a common technique used to detect the presence of IgE in real samples [7,8]. The assay

*Corresponding author (email: ybying@ zju.edu.cn) replaces the plastic binding matrix with a nitrocellulose membrane (NC membrane) with higher adsorption qualities, and therefore, facilitates the binding of larger quantities of target proteins $[9,10]$. Consequently, the dot-blot ELISA possesses higher sensitivity. In addition, since it does not require highly trained technicians and expensive equipment, this detection method can be used for point-of-care testing and on-spot monitoring in clinical, environmental and industrial analysis [11,12]. The traditional dot-blot ELISA used for the detection of IgE employs an antibody as the bio-recognition element. The antibody based assay; however, suffers from some limitations due to the inherent disadvantages of antibodies, such as instability to harsh conditions, limited modifications of the protein and selection in a biological system [13]. Therefore, new bio-recognition elements are highly desirable to explore novel dot-blot assays for the detection of $\mathrm{IgE}$ with enhanced performance.

Recently, aptamers have proven to be efficient alternative receptors to antibodies and have been developed as molecular tools in the field of analytical chemistry. Aptamers, which are selected from large composite oligonu- 
cleotide libraries by an in-vitro evolution process named SELEX (systematic evolution of ligands by exponential enrichment) and readily synthesized in accordance with their reported sequences [14,15], are short single-stranded oligonucleotide ligands that bind targets with high affinity and specificity, because of their specific base sequences and steric configuration [16]. Compared with antibodies, aptamers possess some favorable advantages, such as high affinity, chemical stability and high flexibility. Moreover, they can be easily modified and controlled without obvious deactivation $[17,18]$. Since the discovery of an aptamer of IgE by Wiegand et al. [19] in 1996, several aptamer based assay methods have been developed to detect IgE [20-22]. However, an aptamer based dot-blot assay as a rapid and inexpensive method for the detection of $\operatorname{IgE}$ has not been reported previously.

In this study, a novel dot-blot method for the detection of IgE using an aptamer as the bio-recognition element was developed. The NC membrane was employed as the substrate for blotting the IgE. First, the biotinylated aptamers specifically interacted with $\operatorname{IgE}$ which was immobilized onto the NC membrane (Figure 1). Avidin conjugated horseradish peroxidase (HRP) then bound the biotinylated aptamers via the biotin-avidin interaction. Finally, 3,3',5,5'tetramethylbenzidine (TMB) was catalyzed by the HRP to generate a color change. The spot intensity caused by the color change was found to be proportional to the concentration of IgE. Experimental results demonstrate that the developed method could offer high sensitivity and specificity for the determination of $\mathrm{IgE}$ concentrations.

\section{Experimental}

\subsection{Reagents}

The 5'-biotinylated anti-IgE aptamer was synthesized by Sangon Biotechnology Co., Ltd (Shanghai, China), and its base sequence is: 5'-biotin-GGGGCACGTTTATCCGTC CCTCCTAGTGGCGTGCCCC-3'. Human IgE was purchased from Abcam (Cambridge, UK). Casein, thrombin and immunoglobulin $\mathrm{G}$ ( $\mathrm{IgG})$ were purchased from Sigma-Aldrich (St. Louis, MO, USA). Non-protein blocking reagent, TMB and bovine serum albumin (BSA) were obtained from Sangon Biotechnology Co., Ltd (Shanghai, China). Instant skimmed milk powder was purchased from Boster (Wuhan, China). The avidin conjugated horseradish peroxidase (avidin-HRP) was obtained from Cusabio Biotech Co., Ltd (Wuhan, China). The nitrocellulose membrane (NC membrane) with pores of $0.45 \mu \mathrm{m}$ was purchased from Bio-Rad (Hercules, CA, USA). Other chemicals were reagent grade and were used without further purification. All solutions were prepared with $18.2 \mathrm{M} \Omega \mathrm{cm}$ resistivity deionized water (Millipore, Bedford, MA, USA).

The IgE was dissolved in a $0.01 \mathrm{~mol} / \mathrm{L}$ phosphate buffer solution (PBS, pH 7.4). The aptamer was dissolved in the binding buffer $(20 \mathrm{mmol} / \mathrm{L}$ Tris, $140 \mathrm{mmol} / \mathrm{L} \mathrm{NaCl}, 5$ $\mathrm{mmol} / \mathrm{L} \mathrm{KCl}, 1 \mathrm{mmol} / \mathrm{L} \mathrm{CaCl}_{2}, 1 \mathrm{mmol} / \mathrm{L} \mathrm{MgCl}_{2}, \mathrm{pH}$ 7.4). The solution of aptamers was heated at $95^{\circ} \mathrm{C}$ for $10 \mathrm{~min}$ and gradually cooled to room temperature for $30 \mathrm{~min}$. The thermal treatment disrupts any pre-existing higher order structures that may interfere with the immobilization process. This thermal treatment therefore allows biotin at the $5^{\prime}$ end to interact freely with avidin and promotes aptamer refolding for improved binding efficiency [23].

\subsection{Instrument}

Assays strips were scanned using an ESEQuant lateral flow reader (QIAGEN, CA, USA). The test dot intensity was analyzed using the supporting ESEQuant LF studio software. Analytical parameters are: peak area at fixed baseline and range tolerances $5 \mathrm{~mm}$ left and $5 \mathrm{~mm}$ right of the test line center location.

\subsection{Aptamer based dot-blot assay}

Five microliters of IgE solutions at different concentrations were spotted onto the surface of the NC membrane. When the strip dried, it was immersed into the blocking solution to block the margined sites for $1 \mathrm{~h}$ at room temperature. Excess blocking agent was removed by rinsing with PBST (PBS containing $0.05 \%$ Tween 20) washing buffer for $2 \mathrm{~min}$. Then, the $5 \mu \mathrm{L}$ of the biotinylated aptamers were added onto the membrane for each dot to bind the IgE for $1 \mathrm{~h}$ in a hu-

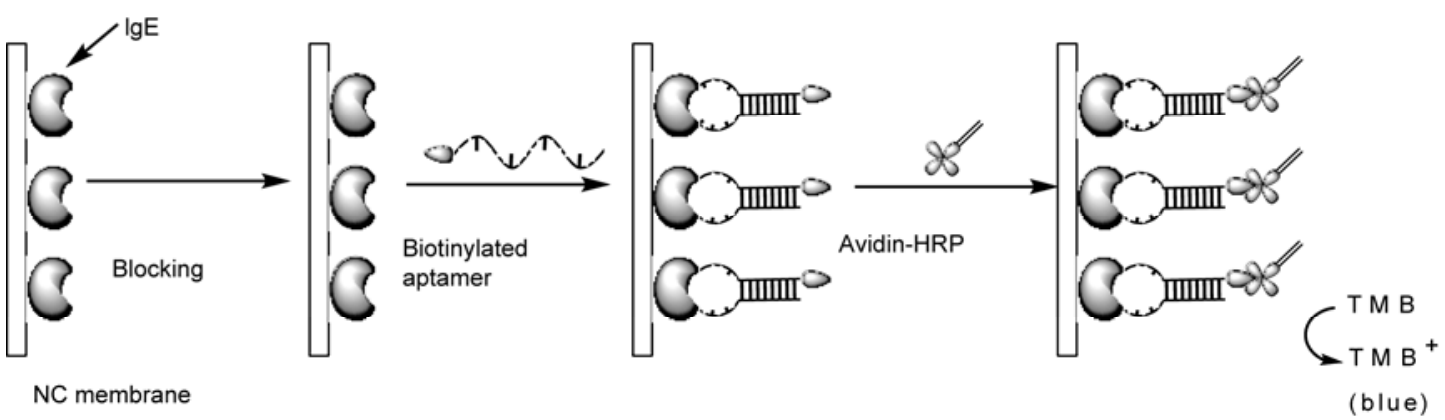

Figure 1 Schematic of the aptamer based dot-blot method for the detection of IgE. 
midified chamber. After rinsing the strip with the PBST washing buffer three times (10 min every washing time), 5 $\mu \mathrm{L}$ of avidin-HRP was dropped onto the membrane at each dot. The strip was washed four times to remove the unbound avidin-HRP and then the fresh TMB- $\mathrm{H}_{2} \mathrm{O}_{2}$ substrate solution was added onto the membrane in the dark for color development. Blue dots emerged within seconds and the intensity of these dots increased over $15 \mathrm{~min}$. Finally the strip was rinsed with deionized water and an image of the dried strip was acquired by a digital camera.

\section{Results and discussion}

\subsection{Characterization of the aptamer based dot-blot assay}

The detection principle is shown in Figure 1. First IgE was immobilized onto the NC membrane by electrostatic and hydrophobic interactions between the protein and the $\mathrm{NC}$ membrane [24]. The NC membrane provides a binding capacity of $80-100 \mu \mathrm{g} / \mathrm{cm}^{2}$ of protein. The margin sites on the membrane were blocked according to a similar interaction. After cleaning the strip with the washing buffer, the biotinylated aptamers were added onto the dots to bind IgE. The biotinylated aptamers recognized $\operatorname{IgE}$ immobilized on the membrane. After washing, the avidin-HRP was blotted onto the membrane to bind the biotinylated aptamers via the biotin-avidin interaction. The biotin-avidin interaction possesses high specificity and the highest affinity constant reported $\left(10^{15} \mathrm{~L} / \mathrm{mol}\right)$, about $10^{3}-10^{6}$ times greater than an interaction between an antigen and antibody [25]. Due to the function of the biotin-avidin system, the colorimetric signal was visibly amplified. Thus the introduced avidin-biotin system has effectively improved the sensitivity of the method for the detection of IgE. Finally, the unbound avidin-HRP was removed by washing carefully and then the fresh TMB- $\mathrm{H}_{2} \mathrm{O}_{2}$ substrate buffer was added. The avidinHRP transferred and amplified the binding event to color signals due to the catalytic activity of HRP. The blue oxidation product of TMB is a cationic free radical and insoluble in water, which may explain why it can be absorbed onto the membrane to give the blue dots [26]. These blue dots emerged in seconds and intensified over $15 \mathrm{~min}$. The intensity of these dots provided a direct measure of the IgE con- centration.

\subsection{Optimization of the reaction conditions}

Several parameters were optimized systematically for the detection of the target protein IgE, including the blocking reagent, the concentration of the biotinylated aptamers and the avidin-HRP.

(i) Blocking reagent. Due to the few antecedents in using aptamer reagents for dot blot assays, the procedure needed to be optimized in terms of surface blocking. The efficiency of the blocking process is extremely important as it reduces the "noise" in the final product, thereby leading to clearer results and the elimination of false positives. The $1 \%$ non-protein blocking reagent, 5\% skim milk, 5\% BSA, 5\% casein and a mix of $5 \%$ casein and $1 \%$ non-protein blocking reagent in Tris-buffered saline (TBS) were used to block the excess sites on the membrane. As shown in Figure 2, the four spots of $1 \%$ non-protein blocking reagent, 5\% skim milk, $5 \%$ BSA and $5 \%$ casein on the strip were colored blue to varying degrees, indicating non-specific adsorption. The largest degree of non-specific binding was obtained when using 5\% skimmed milk as the blocking reagent. This is probably because of the skimmed milk containing small amounts of endogenous biotin residues which caused a high background signal when there was a biotin-avidin system in the assay [27]. In contrast, the mix of $5 \%$ casein and $1 \%$ non-protein blocking reagent eliminated virtually all non-specific background binding, thus this mixture served as the blocking agent in the following experiments.

(ii) Concentration of the biotinylated aptamers and the avidin-HRP. The amount of the biotinylated aptamers plays an important role in the analytical performance of the method for the detection of IgE. A range of quantities of the biotinylated aptamers $(0.5,1,2.5,5,7.5$ and $10 \mu \mathrm{mol} / \mathrm{L})$ was investigated. In the dot-blot assay, the appearance of color at all biotinylated aptamers concentrations examined was observed. As expected, the concentration of the biotinylated aptamers was proportional to the spot intensity (shown as Figure 3(a)). The spot intensity of 1007.42 a.u. was obtained by using $5 \mu \mathrm{mol} / \mathrm{L}$ biotinylated aptamers, whereas a small increase in spot intensity (1097.23 a.u.) was observed when using $10 \mu \mathrm{mol} / \mathrm{L}$ biotinylated aptamers. Here, $5 \mu \mathrm{mol} / \mathrm{L}$ biotinylated aptamers were considered to be
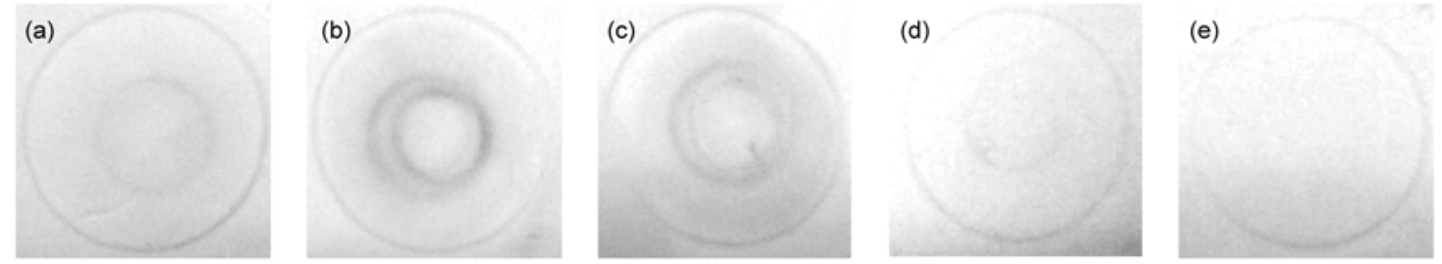

Figure 2 Dot photos for the detection of IgE using different reagents for the blocking. The blocking reagents using for the corresponding spots. (a) $1 \%$ non-protein blocking reagent, (b) 5\% skim milk, (c) 5\% BSA, (d) 5\% casein, and (e) the mix of 5\% casein and $1 \%$ non-protein blocking reagent. The concentration of $\operatorname{IgE}$ was $1 \mu \mathrm{mol} / \mathrm{L}$ for each spot. 

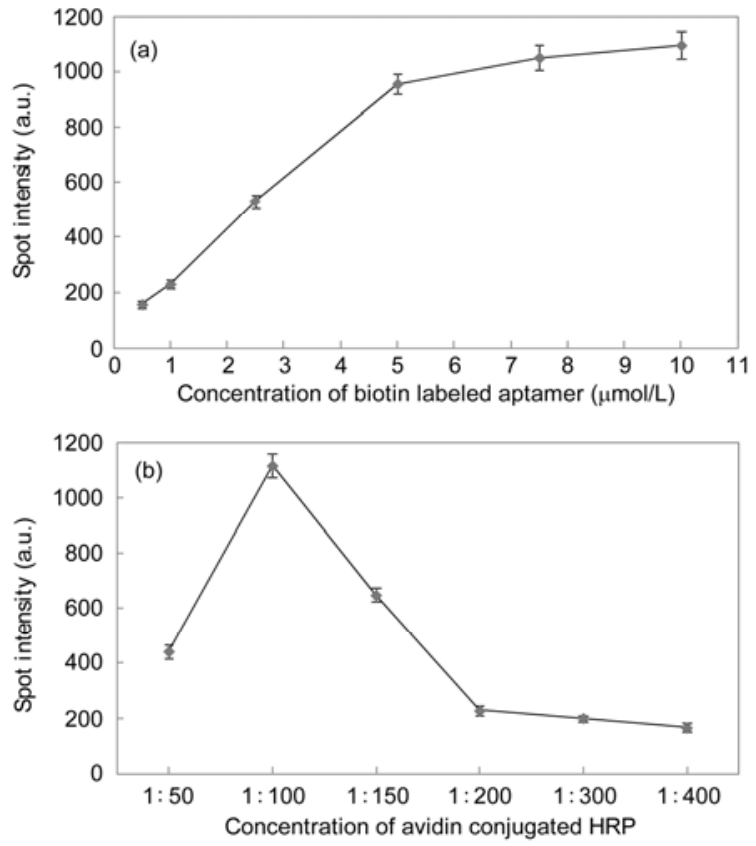

Figure 3 (a) Spot intensity of the colored spot using different concentrations of the biotinylated aptamers $(0.5,1,2.5,5,7.5$ and $10 \mu \mathrm{mol} / \mathrm{L})$. (b) Spot intensity of the colored spots using different concentrations of the avidin-HRP $(1: 400,1: 300,1: 200,1: 150,1: 100$, and $1: 50)$.

adequate and selected as the optimal concentration in the following experiments.

The concentration of the avidin-HRP used in the assay was investigated to find the optimal concentration for the detection of IgE. Different concentrations of the avidin$\operatorname{HRP}(1: 400,1: 300,1: 200,1: 150,1: 100$, and $1: 50)$ were employed. As shown in Figure 3(b), the spot intensity was observed to initially increase with increasing dilution of the avidin-HRP, reaching a maximum at a 100 -fold dilution of the avidin-HRP. Further increases in the dilution of the avidin-HRP to a 400-fold dilution caused a decrease in the spot intensity. Thus, a 100-fold dilution of the avidin-HRP was selected as the optimal concentration of the avidin-HRP for subsequent experiments.

(iii) Analytical performance. The efficiency and minimal detection limit were analyzed using the freshly prepared aptamer based dot-blot assay performed under the optimal reaction conditions, including the mix of $5 \%$ casein and $1 \%$ non-protein blocking reagent as the blocking reagent, 5 $\mu \mathrm{mol} / \mathrm{L}$ biotinylated aptamers, and avidin-HRP at a dilution ratio of 1:100. A series of IgE concentrations were quantified (i.e. $1000,500,250,100,75,50$ and $10 \mathrm{nmol} / \mathrm{L}$ ) in the dot-blot assay. PBS was used as the negative control. As shown in Figure 4(A), the final result was visibly observed. The blue color of these spots darkened with increasing concentrations of IgE. When the level of $\operatorname{IgE}$ was above 10 $\mathrm{nmol} / \mathrm{L}$, it could be effortlessly distinguished by the naked eye. The colorimetric signal reached a saturated state when the concentration of $\operatorname{IgE}$ was $>1 \mu \mathrm{mol} / \mathrm{L}$, and thus there would be no distinct differences in overloaded protein samples. By careful observation, we found that the color in the center of the dots was fainter than on the borders. This observation may be attributed to the diffusion of $\operatorname{IgE}$ from the center to the surrounding area, which was caused by the concentration gradient of $\mathrm{IgE}$ and the pervasion movement of the solution on the NC membrane $[11,26]$.

The spot intensity was quantified by the portable instrument of the ESEQuant lateral flow reader. The plot of the spot intensities as a function of the $\operatorname{IgE}$ concentrations is shown in Figure 4(B). The results demonstrate that the color intensity decreased linearly with decreasing concentration of IgE. The spot intensity of 1000, 500, 250, 100, 75, 50 and 0 nmol/L IgE were 1508.90, 917.24, 434.01, 236.32,

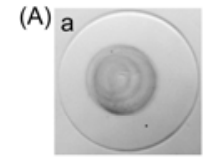

$1 \mu \mathrm{mol} / \mathrm{L}$

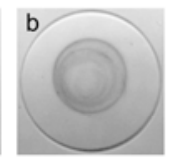

$0.5 \mu \mathrm{mol} / \mathrm{L}$

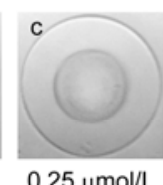

$0.25 \mu \mathrm{mol} / \mathrm{L}$

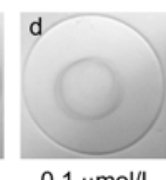

$0.1 \mu \mathrm{mol} / \mathrm{L}$

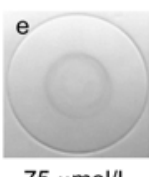

$75 \mu \mathrm{mol} / \mathrm{L}$

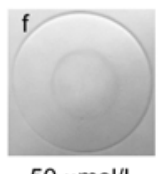

$50 \mu \mathrm{mol} / \mathrm{L}$

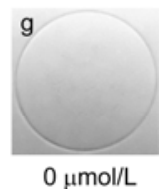

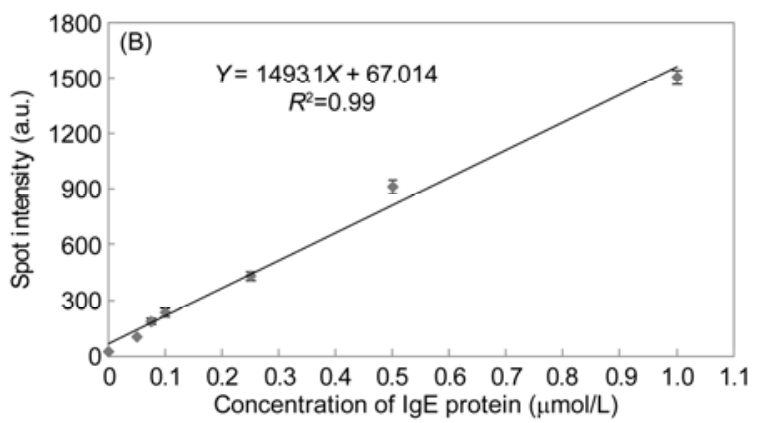

Figure 4 (A) Dot photos for the colorimetric detection of the different concentrations of IgE. (a) 1000, (b) 500, (c) 250, (d) 100, (e) 75, (f) 50, (g) 0 nmol/L). (B) The linear relationship between the spot intensity and the concentrations of IgE in the range of $50 \mathrm{nmol} / \mathrm{L}-1 \mu \mathrm{mol} / \mathrm{L}$. Data were collected from three independent experiments. 
188.07, 106.32 and 27.06 a.u., respectively. A linear response relationship between spot intensity and $\mathrm{IgE}$ concentration in the range from $50 \mathrm{nmol} / \mathrm{L}$ to $1 \mu \mathrm{mol} / \mathrm{L}$ was found. The linear regression equation is $Y=1493.1 X+67.014$ with $R^{2}=0.99$.

The detection limit (LOD) of IgE was calculated as three times the standard deviation for the average measurements of blank samples (LOD $=3 \times \mathrm{SD} / \mathrm{slope}$ ) $[28,29]$. The standard deviation (SD) of the blank sample (zero concentration) was 1.44. Therefore, the LOD was determined to be as low as $2.89 \mathrm{nmol} / \mathrm{L}$, which is lower than the critical concentration defined as being an abnormal level (i.e. $\sim 4 \mathrm{nmol} / \mathrm{L}$ ).

Compared with other methods for the detection of IgE, the aptamer based dot-blot assay has some advantages, including simplicity, rapidity and sensitivity. Due to the strong affinity between the aptamers and proteins, dots can be visible to the naked eye or quantified by a hand-held instrument. The combination of aptamer properties (small size, high affinity and stability) and NC membrane attributes (simple, high adsorption of protein material and low cost) yields an assay that has good specificity and sensitivity, which is suitable for the rapid detection of IgE on-site with high-throughput.

(iv) Specificity. To test the specificity of this assay, three types of proteins, namely BSA, thrombin and IgG, were employed. Among these proteins, IgG has a similar molecular structure to that of $\operatorname{IgE}$ and serves as a good test protein for the aptamer binding specificity. The analytical procedure was the same as the IgE detection method used. The final coloration images are shown in Figure 5. The two spots of $1 \mu \mathrm{mol} / \mathrm{L} \mathrm{BSA}$ and $1 \mu \mathrm{mol} / \mathrm{L}$ thrombin were not colored blue, whereas the spot of $1 \mu \mathrm{mol} / \mathrm{L} \mathrm{IgG}$ was found to color slightly. In contrast, the positive signal from the fourth spot on the right was quite apparent, in which, 1 $\mu \mathrm{mol} / \mathrm{L} \mathrm{IgE}$ was added. The result indicates that the aptamer is specific towards $\operatorname{IgE}$ in this assay.

(v) Stability. The relationship between the scanning time and relative intensity of the spots with $1 \mu \mathrm{mol} / \mathrm{L} \mathrm{IgE}$ was investigated. As shown in Figure 6, the intensity was stable during the scanning time $(0-40 \mathrm{~min})$, and then decreased after $40 \mathrm{~min}$. Therefore, $40 \mathrm{~min}$ was sufficient time for the acquisition of the image and the measurement of the spot intensity. The NC membrane can be kept for a longer period under dark conditions.

\section{Conclusion}

In conclusion, we have developed a simple, rapid and inexpensive dot-blot assay to detect IgE. This assay was based on biotinylated aptamers recognizing $\mathrm{IgE}$ and the color development of avidin-HRP. The aptamer based dot-blot assay for the detection of IgE possesses particular advantages, including high binding affinity on the $\mathrm{NC}$ membrane which allows fast and strong attachment of the protein and the ac-
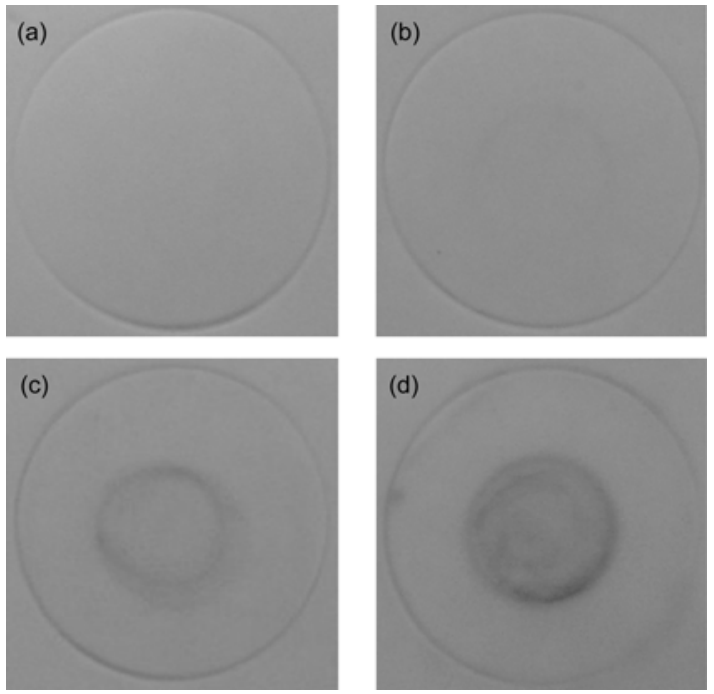

Figure 5 The specificity of the aptamer based dot-blot assay. Dot photos for $5 \mu \mathrm{L}$ of (a) $1 \mu \mathrm{mol} / \mathrm{L} \mathrm{BSA}$, (b) $1 \mu \mathrm{mol} / \mathrm{L}$ thrombin, (c) $1 \mu \mathrm{mol} / \mathrm{L} \mathrm{IgG}$, and (d) $1 \mu \mathrm{mol} / \mathrm{L}$ IgE solutions.

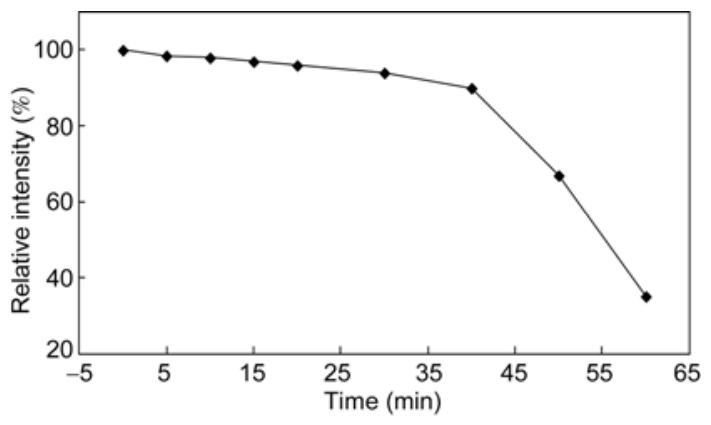

Figure 6 The stability of the aptamer based dot-blot assay.

cumulation of the solid color product over a small area; thereby enhancing the signal intensity. In addition, the result was visibly observable and quantification can be obtained using a simple portable instrument. This is a colorimetric assay for the highly efficient sensing of IgE, and the assay gives excellent analytical performance such as a low detection limit, low cost, considerable time saving and high selectivity.

This work was supported by the National Natural Science Foundation of China (30825027) and Special Fund for Agro-Scientific Research in the Public Interest (200903009).

1 Kim $\mathrm{S}$, Lee $\mathrm{J}$, Lee $\mathrm{S}$ J, et al. Ultra-sensitive detection of $\operatorname{IgE}$ using biofunctionalized nanoparticle-enhanced SPR. Talanta, 2010, 81: 1755-1759

2 Saito H. Much atopy about the skin: Genome-wide molecular analysis of atopic eczema. Int Arch Allergy Immunol, 2005, 137: 319-325

3 Ishizaka K, Ishizaka T. Identification of gammer-antibodies as a carrier of reaginic activity. J Immunol, 1967, 99: 1187-1198

4 Johansson S G, Bennich H. Immunological studies of a typical (myeloma) immunoglobulin. Immunology, 1967, 13: 381-394

5 Platts-Mills T A E. The role of immunoglobulin E in allergy and 
asthma. Am J Respir Crit Care Med, 2001, 164: S1-S5

6 Wide L, Bennich H, Johansso S G. Diagnosis of allergy by an in-vitro test for allergen antibodies. Lancet, 1967, 2: 1105-1107

7 Kirsch S, Fourdrilis S, Dobson R, et al. Quantitative methods for food allergens: A review. Anal Bioanal Chem, 2009, 395: 57-67

8 Wongkamchai S, Vaiyavatjamai P, Wanachiwanawin D, et al. A dot-blot and immuno-blot assay for the detection of mosquito saliva specific IgE in mosquito bite allergic subjects. J Dermatol Sci, 2009 , 53: $155-157$

9 Bargou I, Mankai A, Jamaa A, et al. Detection of M2 antimitochondrial antibodies by dot blot assay is more specific than by enzyme linked immunosorbent assay. Pathol Biol, 2008, 56: 10-14

10 Szucs J, Gyurcsanyi R E. Towards protein assays on paper platforms with potentiometric detection. Electroanalysis, 2012, 24: 146-152

11 Zhu J B, Li T, Hu J M, et al. A novel dot-blot DNAzyme-linked aptamer assay for protein detection. Anal Bioanal Chem, 2010, 397: 2923-2927

12 Courtioux B, Bisser S, M'Belesso P, et al. Dot enzyme-linked immunosorbent assay for more reliable staging of patients with human African trypanosomiasis. J Clin Microbiol, 2005, 43: 4789-4795

13 Wang Y X, Ye Z Z, Si C Y, et al. Application of aptamer based biosensors for detection of pathogenic microorganisms. Chin $\mathrm{J}$ Anal Chem, 2012, 40: 634-642

14 Ellington A D, Szostak J W. In vitro selection of RNA molecules that bind specific ligands. Nature, 1990, 346: 818-822

15 Tuerk C, Gold L. Systematic evolution of ligands by exponential enrichment-RNA ligands to bacteriophage-T4 DNA-polymerase. Science, 1990, 249: 505-510

16 Wang J L, Lü R J, Xu J J, et al. Characterizing the interaction between aptamers and human $\operatorname{IgE}$ by use of surface plasmon resonance. Anal Bioanal Chem, 2008, 390: 1059-1065

17 Tang L H, Liu Y, Ali M M, et al. Colorimetric and ultrasensitive bioassay based on a dual-amplification system using aptamer and DNAzyme. Anal Chem, 2012, 84: 4711-4717

18 Chang H X, Tang L H, Wang Y, et al. Graphene fluorescence reso- nance energy transfer aptasensor for the thrombin detection. Anal Chem, 2010, 82: 2341-2346

19 Wiegand T W, Williams P B, Dreskin S C, et al. High-affinity oligonucleotide ligands to human $\mathrm{IgE}$ inhibit binding to Fc epsilon receptor I. J Immunol, 1996, 157: 221-230

20 Li H, Qiang W B, Vuki M, et al. Fluorescence enhancement of silver nanoparticle hybrid probes and ultrasensitive detection of IgE. Anal Chem, 2011, 83: 8945-8952

21 Tran D T, Vermeeren V, Grieten L, et al. Nanocrystalline diamond impedimetric aptasensor for the label-free detection of human $\operatorname{IgE}$. Biosens Bioelectron, 2011, 26: 2987-2993

22 Kim Y H, Kim J P, Han S J, et al. Aptamer biosensor for lable-free detection of human immunoglobulin $\mathrm{E}$ based on surface plasmon resonance. Sens Actuator B-Chem, 2009, 139: 471-475

23 Tennico Y H, Hutanu D, Koesdjojo M T, et al. On-chip aptamer-based sandwich assay for thrombin detection employing magnetic beads and quantum dots. Anal Chem, 2010, 82: 5591-5597

24 Farrah S R, Shah D O, Ingram L O. Effects of chaotropic and antichaotropic agents on elution of poliovirus adsorbed on membrane filters. Proc Natl Acad Sci USA, 1981, 78: 1229-1232

25 Diamandis E P, Christopoulos T K. The biotin (strept)avidin system-Principles and applications in biotechnology. Clin Chem, 1991, 37: 625-636

26 Li J S, Yao J J, Zhong W W. Membrane blotting for rapid detection of mercury(II) in water. Chem Commun, 2009, 4962-4964

27 Jeon M, Kim J, Paeng K J, et al. Biotin-avidin mediated competitive enzyme-linked immunosorbent assay to detect residues of tetracyclines in milk. Microchem J, 2008, 88: 26-31

28 Kim Y H, Kim J P, Han S J, et al. Aptamer biosensor for lable-free detection of human immunoglobulin $\mathrm{E}$ based on surface plasmon resonance. Sens Actuator B-Chem, 2009, 139: 471-475

29 Cao C, Kim J P, Kim B W, et al. A strategy for sensitivity and specificity enhancements in prostate specific antigen- $\alpha_{1}$-antichymotrypsin detection based on surface plasmon resonance. Biosens Bioelectron, 2006, 21: 2106-2113

Open Access This article is distributed under the terms of the Creative Commons Attribution License which permits any use, distribution, and reproduction in any medium, provided the original author(s) and source are credited. 